\title{
A striking pattern of cortisol non-responsiveness to psychosocial stress in patients with panic disorder with concurrent normal cortisol awakening responses
}

\author{
Katja Petrowski ${ }^{\text {a, }}$, Ulf Herold ${ }^{\text {a }}$, Peter Joraschky ${ }^{\text {a }}$, Hans-Ulrich Wittchen ${ }^{\text {b }}$, Clemens \\ Kirschbaum ${ }^{\text {b }}$ \\ a Institute of Psychotherapy and Psychosomatic Medicine, School of Medicine, Technische \\ Universität Dresden, Fetscherstr. 74, 01307 Dresden, Germany \\ b Department of Psychology, Technische Universität Dresden, Dresden, Germany
}

\section{Summary}

Background: Subtle and inconsistent differences in hypothalamic-pituitary-adrenal (HPA) axis activity have been reported for patients with panic disorder. While these patients show little or no alterations in basal ACTH and cortisol levels, it has been hypothesized that HPA hyperresponsivity was a trait in panic patients when exposed to novel and uncontrollable stimulation.

Methods: Thirty-four patients ( 23 females, mean age 35 yrs) diagnosed with panic disorder were compared to 34 healthy controls matched for age, gender, smoking status, and use of oral contraceptives. Both groups were exposed twice to a potent laboratory stress protocol, the Trier Social Stress Test (TSST) on consecutive days. Free salivary cortisol levels and heart rate responses were repeatedly measured before and following the TSST. In addition, the cortisol awakening response (CAR) was assessed to further investigate HPA reactivity in PD patients.

Results: While the TSST induced similar heart rate stress responses in both groups, cortisol responses were clearly absent in the panic patients with normal responses in the controls $(F(1.96,66)=20.16 ; p<0.001)$. No differences in basal cortisol levels were observed in the extended baseline period. The same cortisol stress non-response patterns were observed when patients with/without comorbid depression, or with/without psychotropic medication were compared. In contrast to their non-response to the psychosocial stressor, panic patients showed a significant CAR.

Conclusion: These findings provide strong evidence to suggest that PD patients present with a striking lack of cortisol responsivity to acute uncontrollable psychosocial stress under laboratory conditions. This unresponsiveness of the HPA axis appears to be rather specific, since a normal CAR in the morning could be documented in these patients. Thus, the present results do not support the hypothesis that PD patients show a trait HPA hyperresponsiveness to novel and uncontrollable stimulation. In contrast, the data provide support for a hyporesponsive HPA axis under emotional stress in PD patients.

Keywords: HPA system; Anxiety disorder; Stress reactivity; Cortisol; Panic disorder; Comorbidity 


\section{Introduction}

Panic disorder is defined as a clinical condition in which the patients experience recurrent episodes of panic attacks and constant concern of future attacks. It is a common disorder with significant burden of suffering for the individual and significant economic costs for the society, respectively (Greenberg et al., 1999). The disease has been linked to increased mortality and cardiovascular disease (Albert et al., 2005; Smoller et al., 2005).

Among a multitude of other bodily systems, considerable research efforts have tried to associate panic disorder with an abnormal functioning of the hypothalamic-pituitary-adrenal system (HPA). While some reports suggested increased basal cortisol production, blunted ACTH and cortisol responses to CRH infusion, along with subtle differences in feedback sensitivity of the axis (Roy-Byrne et al., 1986; Holsboer et al., 1987; Gurguis et al., 1991; Schreiber et al., 1996; Erhardt et al., 2006; Abelson et al., 2007), findings have remained inconsistent throughout the past 20 years. Furthermore, studies of naturally occurring panic attacks showed that these attacks occur without an apparent secretory episode of cortisol inmost instances (Cameron et al., 1987; Bandelow et al., 2000). This eminent lack of cortisol response to a situation often perceived by the patients as life threatening is puzzling, since HPA activity is well known to rapidly increase in times of threat or harmful stimulation (Mason, 1968). However, most patients studied for acute endocrine responses to panic attacks (naturally or in the lab) had experienced dozens of attacks before. Thus, it is tempting to speculate that the cortisol non-response to acute panic attacks simply reflects successful habituation to repeated stimulation by complex emotional events. In healthy volunteers, rapid habituation of cortisol responses can be observed when subjects are repeatedly exposed to stressful stimuli in the same environmental context (Kirschbaum et al., 1995; Schommer et al., 2003). Habituation of cortisol stress responses under such conditions indicate a decrease in novelty, unpredictability, and uncontrollability of the situation.

Summarizing the contradictory and inconsistent findings on HPA abnormalities in patients suffering from panic disorder, Abelson et al. (2007) concluded that this disorder is characterized by an enhanced sensitivity to novelty with a heightened fear of panic in unfamiliar environments. They hypothesized that in response to novel experimental contexts panic disorder patients would therefore display an enhanced HPA reactivity. Unfortunately, the literature on acute ACTH or cortisol responses to such stimuli involving novel, uncontrollable, and threatening experiences is sparse at best. Only three published studies so far investigated HPA responses to psychosocial challenge (Leyton et al., 1996; Hoehn et al., 1997; Garcia-Leal et al., 2005). However, none of the stimulation protocols employed in these studies lead to significant cortisol elevations in either, panic patients or control subjects.

In order to test the hypothesis of a heightened HPA responsiveness to novel, uncontrollable, and threatening stimuli in panic disorder patients, we used a well-established psychosocial stress paradigm, the Trier Social Stress Test (TSST; Kirschbaum et al., 1993). In a recent meta analysis, the TSST has been found to be among the most potent manipulations for the induction of acute cortisol responses under laboratory conditions (Dickerson and Kemeny, 2004). With healthy adolescents or adults, the TSST yields an average responder rate of 75$80 \%$ of all subjects tested. Thus, this protocol appeared well suited for an appropriate investigation of putative HPA response differences in patients with panic disorder. 


\section{Materials and methods}

\subsection{Study participants}

Patients with panic disorderwere recruited from January 2006 to July 2007 at the Carl Gustav Carus University Hospital of the Technische Universitaet Dresden, Germany. The Structured Clinical Interview (SCID, Spitzer et al., 1990; Wittchen et al., 1990) for the Diagnostic and StatisticalManual of Mental Disorders (DSM-IV) was used to diagnose of panic disorder with or without agoraphobia (APA, 2004), respectively.

Patients with a current primary diagnosis of panic disorder with or without agoraphobia were included. A secondary diagnosis of dysthymia or mild depression did not preclude participation. Diagnostic exclusion criteria were: Any other mental disorders according to the SCID, and any acute and/or chronic medical illness as assessed by a physical examination and routine laboratory. In order to avoid a significant selection bias in the recruitment of patients, habitual cigarette smoking, use of oral contraceptives, or pharmaceutical treatments of panic disorder were allowed. They were carefully matched on these variables with healthy control subjects (except for the use of pharmaceutical drug treatment). A total of 47 patients were approached. Twelve patients did not meet the inclusion criteria, and one patient dropped out during the procedure. Of the 12 patients that did not meet the inclusion criteria, five did not fulfill the SCID criteria for panic disorder, five suffered additionally from somatoform disorders, one was found to have additionally a post-traumatic stress disorder and one was excluded due to drug abuse (Fig. 1).

The final study sample consisted of $\mathrm{N}=34$ patients with a primary diagnosis of panic disorder, 11 patients of whom were diagnosed with a secondary diagnosis of depression. The mean age at the onset of panic disorder was $29.00(\mathrm{SD}=11.09)$ years of age, and the mean duration of the panic disorder was $6.06(\mathrm{SD}=8.00)$ years. According to the Panic and Agoraphobia-Severity-Scale (PAS) (Bandelow, 1979) most of the panic symptoms were rated as severe.

Of the 34 patients, 19 were on various drug treatments at the time of testing [selective serotonin reuptake inhibitors (SSRIs; $\mathrm{n}=5$ ), serotonin norepinephrine reuptake inhibitors (SNRIs; $n=5)$, tricyclic antidepressants $(n=2)$, tetracyclic antidepressants $(n=3)$, buspiron (anxiolytic; $\mathrm{n}=1$ ), monoamine oxidase inhibitors (MAOIs; $\mathrm{n}=1$ ), atypical antidepressants $(\mathrm{n}$ $=1)$, phytosedatives $(n=4)]$.

The healthy controls $(n=34)$ had been recruited through newspaper advertisements, and were matched by age and gender to the patient sample. Out of 67 volunteers, twelve were excluded due to a current or life-time major mental disorder revealed by the SCID. From the remaining 55 volunteers, 34 were selected to provide close matches to the 34 patients with respect to age and gender. Characteristics of patients and matching controls are provided in Table 1.

All study participants provided written informed consent. The study protocol was approved by the local Ethics Committee of the Medical Faculty of the Technische Universität Dresden, Germany (No EK7012006).

\subsection{Procedures}

The participants were individually scheduled for the TSST sessions on 2 consecutive days ( $\mathrm{t}$, t2) between $0300 \mathrm{~h}$ and $1800 \mathrm{~h}$ in order to minimize the circadian variations in cortisol levels. 
Upon arrival at the laboratory, subjects were fitted a belt for continuous wireless transmission of heart rate signals (Polar S810, Polar, Finland). Following a brief baseline period of 25 min, the study participants were exposed to the TSST. This psychosocial stress protocol mainly consists of a social-evaluative situation including a 5 min public speaking task and a subsequent 5 min mental arithmetic task in front of a two-person panel. A detailed description and evaluation of the TSST was recently published (Kudielka et al., 2007 — The TSST revisited: Ten years of research). The participants were asked to refrain from eating, drinking, and smoking for at least $2 \mathrm{~h}$ before testing as well as during the one and a half hour testing session. Healthy controls and patients were tested in the same setting with identical procedures and staff present at each of the two repeated TSST sessions. A total of seven saliva samples were collected throughout each of the two TSST-sessions (10 min before, 1, 10, 20, 30, 40, and $50 \mathrm{~min}$ after completion of the TSST). In addition to the TSST, patients collected two saliva samples on 2 consecutive weekday mornings immediately at wake-up, and $30 \mathrm{~min}$ thereafter. Approximately $70-80 \%$ of all subjects tested so far show a marked cortisol increase of $50-80 \%$ half an hour after wake-up. This response has been termed ,cortisol awakening response“ (CAR; Fries, Dettenborn and Kirschbaum, 2008). Compliance with the saliva collection protocol was electronically monitored (MEMs caps, Aardex, Switzerland).

\subsection{Saliva collection and cortisol analysis}

For quick and hygienic collection of saliva samples Salivette swabs were used (Sarstedt, Nümbrecht, Germany). The samples were kept frozen at $-20{ }^{\circ} \mathrm{C}$ until assay. Before analysis, samples were centrifuged at $3000 \mathrm{rpm}$ for $5 \mathrm{~min}$ to produce a clear supernatant of low viscosity. $50 \mathrm{ml}$ were removed for cortisol analysis using a commercially available immunoassay with chemiluminescence detection. The lower detection limit of this assay is $0.43 \mathrm{nmol} / \mathrm{l}$. Intra- and inter-assay coefficients of variation were below $8 \%$ for low $(3 \mathrm{nmol} / \mathrm{l})$ and high $(25 \mathrm{nmol} / \mathrm{l})$ cortisol levels, respectively.

\subsection{Psychopathological assessments}

In patients, the psychopathological burden and severity of panic-agoraphobia wasmeasured by two instruments before the patients' psychotherapeutic treatment: (1) The Symptom Check List (Derogatis, 1977; Franke, 2002) consisting of 90 items with a five-point rating scale (total score range 20-80) for the patient to evaluate his/her own psychological and physical impairment. In addition, (2) the Panic and Agoraphobia Severity Scale (Bandelow, 1979) was used to assess the specific symptom severity for anxiety and phobic anxiety. This self-rating questionnaire consists of 13 items rated on a five-point rating scale (total score range: $0-52$ ). The depressive symptoms were evaluated by (3) the Beck Depression Inventory (BDI) (Beck et al., 1961; Hautzinger et al., 1994). The BDI consists of 21 symptoms and attitudes which are rated in terms of intensity from 0 to 3 (total score range: $0-63$ ).

\subsection{Statistical analysis}

Analyses of variance (ANOVAs) for repeated measures were performed to reveal possible main or interaction effects in cortisol and heart rate responses to the TSST for panic patients and healthy control subjects. Greenhouse-Geisser correction was applied to account for violation of the sphericity assumption. A response to the TSST was defined as an increase in cortisol levels of $2.5 \mathrm{nmol} / \mathrm{l}$ over the baseline (Van Cauter and Refetoff, 1985). Main effects of smoking, use of oral contraceptive medication, and psychotropic drugs were tested by univariate ANOVAs. The CAR on the two consecutive mornings was tested for statistical 
significance by ANOVA for repeated measures. Data analyses were performed using SPSS v. 12 (SPSS Inc., Chicago, IL, USA).

\section{Results}

There were no significant differences in baseline cortisol levels between panic disorder patients and healthy controls before either of the two TSST sessions (both $\mathrm{F}<1$ ). Also, baseline levels were not influenced by smoking status, use of contraceptive pills, or psychotropic medication status, respectively (all $\mathrm{p}<.01$ ). While TSST exposure lead to the well-documented increase in cortisol levels in healthy controls (responder rates: $82 \% \mathrm{t} 1 ; 59 \%$ t2), PD patients failed to show a significant elevation of cortisol levels on either test day (responder rates: $8.8 \% \mathrm{t} 1 ; 2.9 \% \mathrm{t} 2$; chi-square tests for responder rates in both groups: $\mathrm{t} 1$ : Chi2 = 19.33, df = 1, p <.001; t2: Chi2 = 12.21, df =1, p <.001); see Fig. 2(a)— (b)). ANOVA results indicated significant main group effect $(\mathrm{F}(1.66)=8.56 ; \mathrm{p}<.001)$ as well as a significant group by time interaction effect for both test days $(\mathrm{F} 1.95,66=12.67 ; \mathrm{p}<.001)$. While healthy controls showed a small habituation effect with lower cortisol responses upon the second stress exposure $(\mathrm{F} 2.33,33=12.13 ; \mathrm{p}<.001)$, no differences in cortisol patterns were observed in the patient group across days $(\mathrm{F}<1)$. Within the group of PD patients, neither a secondary diagnosis of depression, nor use of psychotropic medication showed an impact on the cortisol patterns (all F $<1$; see Fig. 2(c) and (d)). Likewise, neither smoking nor use of oral contraceptives had an impact on the cortisol response pattern in the PD patients. Separate analyses with either only nonsmokers, or women without oral contraceptives revealed the same result. No significant cortisol responses were observed in these subgroups of patients either (Fig. 2c, d).

While the PD patients did not respond with elevated cortisol levels in response to the psychosocial stress test, they displayed a significant rise of cortisol levels after awakening on both days tested $(\mathrm{F} 1,28=16.13, \mathrm{p}<.001)$ with no significant difference in CAR magnitude between the two consecutive mornings $(\mathrm{F} 1,28<0.01, \mathrm{p}=.97)$. In contrast to the cortisol response patterns, heart rate responses did not differ between PD patients and controls (Fig. 3). Significant heart rate increases were observed in both groups for both TSST sessions with no response attenuation to the second TSST exposure $(\mathrm{F} 2.82,66=1.75 ; \mathrm{p}=.17)$.

\section{Discussion}

This is the first report of a striking cortisol non-response to moderate or intense psychosocial stress in a group of patients with panic disorder. Although such a hypo/non-response pattern had been suggested by other studies in the past (Leyton et al., 1996; Hoehn et al., 1997; Garcia-Leal et al., 2005), these experiments yielded inconclusive data due to stress protocols that did not lead to HPA activation in the control subjects either. In the present work, we employed a well-established potent laboratory stress protocol, the TSST, for significant activation of the HPA axis. As expected, control subjects showed a typical TSST-induced rise in cortisol levels with a peak 10 min following stress cessation. With some $80 \%$ of controls showing a rise of at least $2.5 \mathrm{nmol} / \mathrm{l}$ in response to the TSST, also the percentage of cortisol stress responders was in the typical range (Kirschbaum, Pirke and Hellhammer, 1993). In contrast to these individuals, less than 10\% PD patients showed a cortisol rise indicative of a secretory episode to the first TSST, while only a single PD patient had a cortisol response to the second TSST. With an experience of more than 15 years of stress research with the TSST, this laboratory has never before observed such a uniform pattern of cortisol nonresponsiveness in any group of subjects or patients investigated. Moreover, the TSST nonresponsiveness with regard to free cortisol levels cannot be explained by the use of 
psychotropic medication, nor with comorbid depression. In fact, if any, only those PD patients with comorbid depression tended to show a small (not statistically significant) elevation of cortisol levels. Likewise, the use of oral contraceptives or habitual cigarette smoking does not differentiate between the two groups of adults tested here either. In the controls, similar numbers of subjects smoked and/or used oral contraceptives. Despite a trend towards lower responses, these subjects clearly responded to the TSST. This was not the case in the group of PD patients.

Our results suggest a rather specific failure to mount a significant cortisol response to egothreatening, uncontrollable psychosocial stress in PD, since the patients showed a completely normal cortisol rise $30 \mathrm{~min}$ after waking up in the morning (CAR). As suggested by previous work, the CAR correlates well with the adrenal capacity to respond to ACTH stimulation, but not with the individual TSST response (Schmidt-Reinwald et al., 1999). Thus, in principal the adrenal cortex of PD patients appears to be responsive to ACTH, however, there is a clear lack of $\mathrm{CRH}$ or ACTH signaling to the adrenal cortex in response to distressing psychosocial stimulation. The present data therefore contradict conclusions drawn by Abelson et al. (2007) that PD patients would show an enhanced HPA responsiveness to novel and uncontrollable situations.

Where does this apparent stress non-responsiveness come from? One might speculate that the current findings could suggest that PD patients respond to ego-threatening uncontrollable stress with dissociation. While this appears to be an attractive speculation, the heart rate responses of our patients are clearly indicative that they actively and consciously participated in the TSST tasks.

Alternatively, one may think of habituation as a proper explanation for the cortisol nonresponse pattern observed here. Most patients with panic disorder are prone to experience a large number of panic attacks over the course of years. It could be argued, that consistent with McEwen's pattern of „repeated hits“ in his allostatic load model (1998), these patients develop such non-responsiveness over time. The many instances that the patients experience a situation, which they perceive as life threatening, might be related to an absent HPA response to a 'minor' stressor like the TSST. Following this line of reasoning, we have begun to investigate newly diagnosed PD patients who have had one or two panic attacks only. None of these patients mounted a cortisol response to the TSST either (data not shown). In addition, successful psychotherapy (with or without psychotropic medication) did not appear to reverse the pattern of cortisol nonresponsiveness to the TSST in our patients (data not shown). Although inconclusive for now (due to a small number of patients studied so far), the present observations in PD patients speak for a characteristic trait. An exciting hypothesis might thus be to speculate that a pattern of HPA (or cortisol) non-responsiveness to psychosocial stress represents a vulnerability factor for a later development of PD. Either causally or just correlationally related, such risk factor might be associated with a specific genotype pattern (Smoller et al., 2008). Genetic polymorphisms that are associated with an increased processing of emotional stimuli in the prefrontal cortex, such as the COMT val158met polymorphism (Domschke et al., 2008), might be involved in the downregulation of the HPA response to stressful stimuli. Such inverse correlation between activity in the prefrontal cortex and cortisol response to psychosocial stress has been documented in functional imaging studies recently (Wang et al., 2005; Kern et al., 2008). To what extent this apparent cortisol non-responsiveness to psychosocial stress is linked to the increased mortality and cardiovascular disease observed in these patients (Albert et al., 2005; Smoller et al., 2005), remains to be elucidated. 
While the present results may unveil an interesting psychoneuroendocrine feature of PD patients, our findings need to be replicated in a study with concurrent saliva and blood samples. The present work was performed only with salivary cortisol measures - which might be the relevant fraction of cortisol to reach the target tissues, including the brain (Hellhammer et al., 2009). However, additional ACTH and total cortisol measures would describe in more detail to what extent and at which level the HPA axis is disturbed or dysfunctional in PD patients.

Table 1 Patient characteristics, subsamples of patients and matching of controls.

\begin{tabular}{|c|c|c|c|c|c|c|c|c|}
\hline & \multirow{2}{*}{$\begin{array}{l}\text { Total sample } \\
\text { Patients }\end{array}$} & \multirow[t]{2}{*}{ Controls } & \multirow[t]{2}{*}{$t / x^{2}$} & \multirow[t]{2}{*}{$P$} & \multicolumn{3}{|c|}{$\begin{array}{l}\text { Subsamples of panic disorder (PD) } \\
\text { patients }\end{array}$} & \multirow[t]{2}{*}{$P$} \\
\hline & & & & & Pure PD & $\begin{array}{l}\text { PD With secondary } \\
\text { depression }\end{array}$ & $t / \chi^{2}$ & \\
\hline Total (N) & 34 & 34 & & & 23 & 11 & & \\
\hline Females (n) & 23 & 23 & & & 16 & 7 & & \\
\hline Males (n) & 11 & 11 & & & 7 & 4 & 0.12 & $.99^{\dagger}$ \\
\hline Age (years), mean (S.D.) & $35.0(12.0)$ & $35.3(12.2)$ & -0.08 & $.94^{*}$ & $34.3(12.8)$ & $36.6(10.3)$ & -0.54 & $.60^{\circ}$ \\
\hline Smokers (n) & 15 & 8 & 3.22 & $.12^{\dagger}$ & 9 & 6 & 0.72 & $.48^{\dagger}$ \\
\hline Oral contraceptives (n) & 12 & 7 & 2.24 & $.23^{\dagger}$ & 8 & 4 & 0.10 & $.99^{\dagger}$ \\
\hline Psychotropic drug treatment (n) & 17 & 0 & & & 11 & 8 & 1.87 & $.27^{\dagger}$ \\
\hline BDi, mean (S.D.) & $15.3(8.0)$ & & & & $12.9(7.6)$ & $19.6(6.8)$ & -2.44 & $.02^{*}$ \\
\hline SCL GSI, mean (S.D.) & $69.1(11.2)$ & & & & $65.7(11.3)$ & $75.3(8.37)$ & -2.46 & $.02^{*}$ \\
\hline PAS, mean (S.D.) & $20.3(12.6)$ & & & & $16.3(9.4)$ & $30.2(14.5)$ & -3.00 & $.01^{*}$ \\
\hline
\end{tabular}

't-test; 'Fisher's exact test; BDI, beck-depression-invertory; SCL, symptom check list; GSI, general-symptom-index; PAS, panic and agorap hobia-severity-scale.

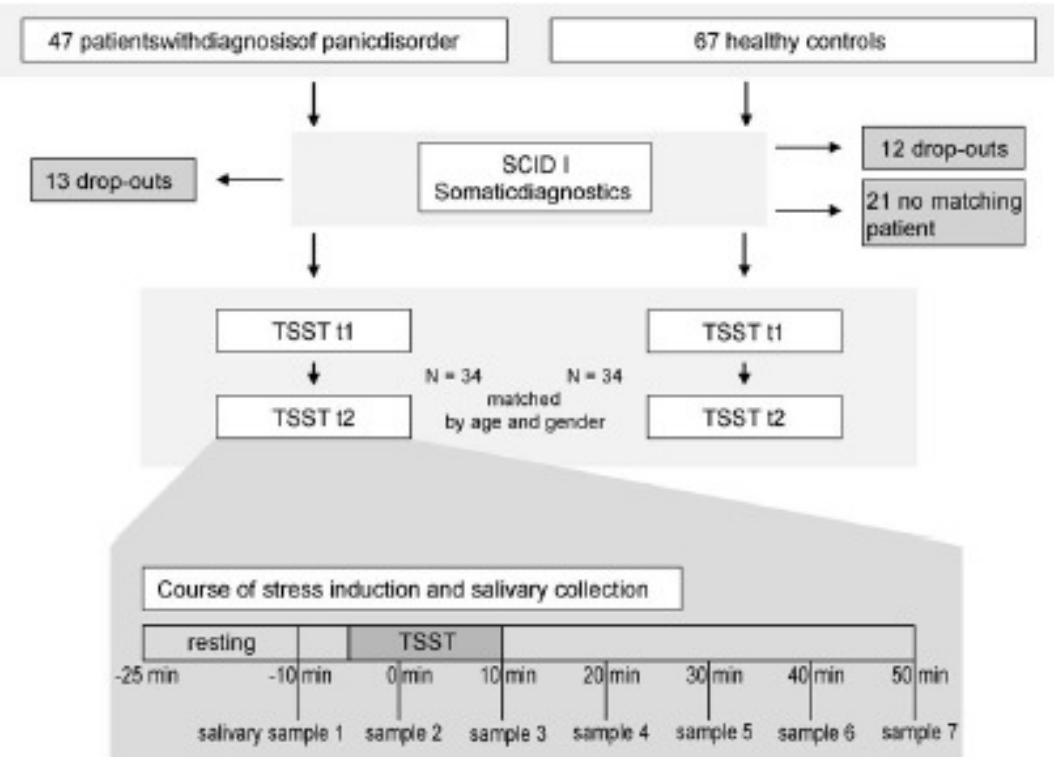

Figure 1 Flow chart of the selection of patients and healthy individuals, the study design with the Trier Social Stress Test, and the time intervals used for salivary sample probes. 

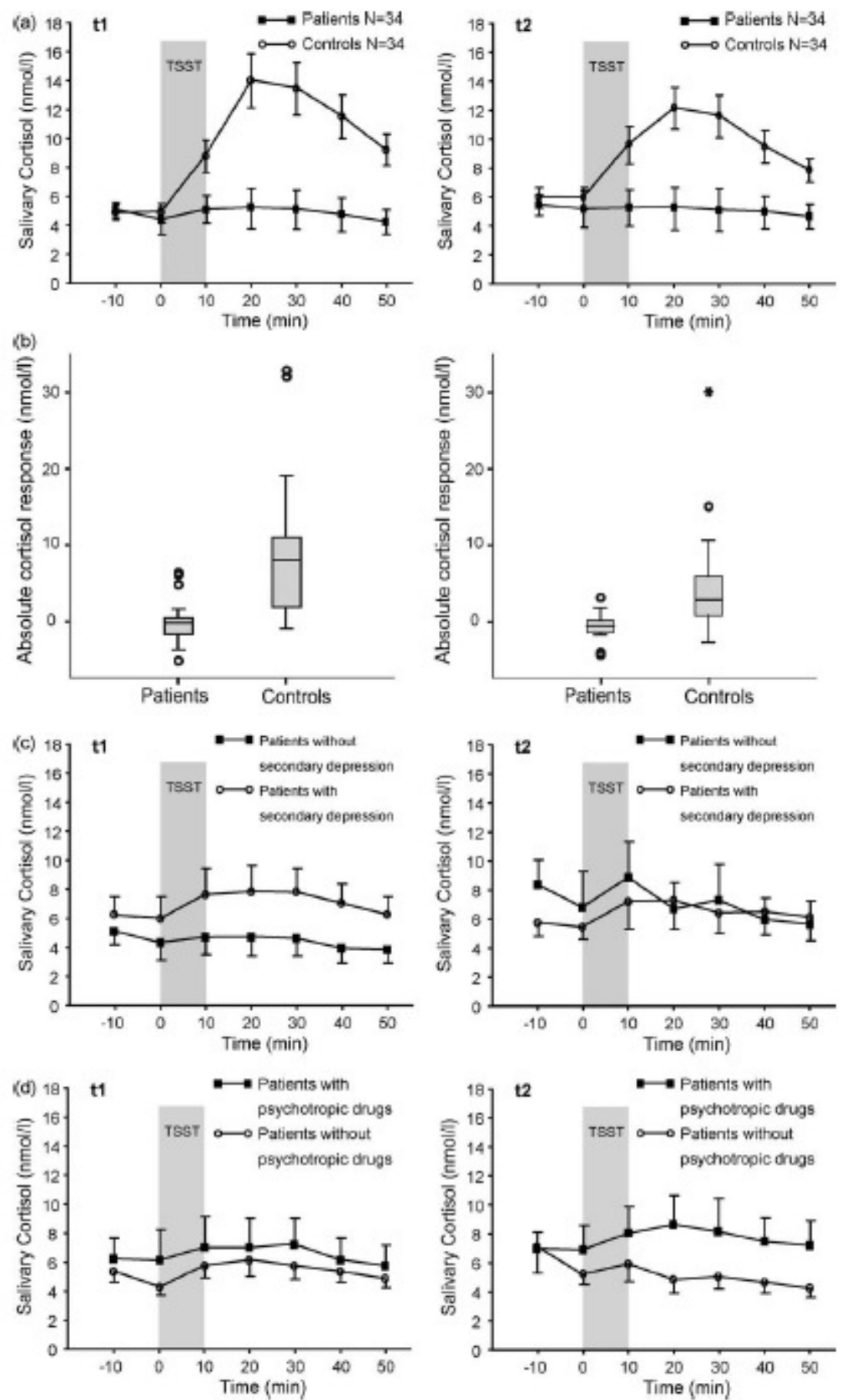

Figure 2 (a) Cortisol responses to psychosocial stress (TSST) in patients with panic disorder and healthy matched control subjects on 2 consecutive days ( $\mathrm{t} 1, \mathrm{t} 2$ ). (b) Boxplots of the absolute cortisol response (maximum cortisol level after TSST minus baseline) to psychosocial stres (TSST) in patients with panic disorder and healthy controts subjects on 2 consecutive days (t1, t2). (c) Cortisol responses to psychosocial stress (TSST) on 2 consecutive days ( 1 1, t2) in patients with a pure panic disorder, and patients with a panic disorder and a secondary diagnosis of depression, respectively. (d) Cortisol responses to psychosocial stress (TSST) in patients with and without psycho-pharmaceutical treatment of the panic disorder on 2 consecutive days (t1, $t 2)$. 

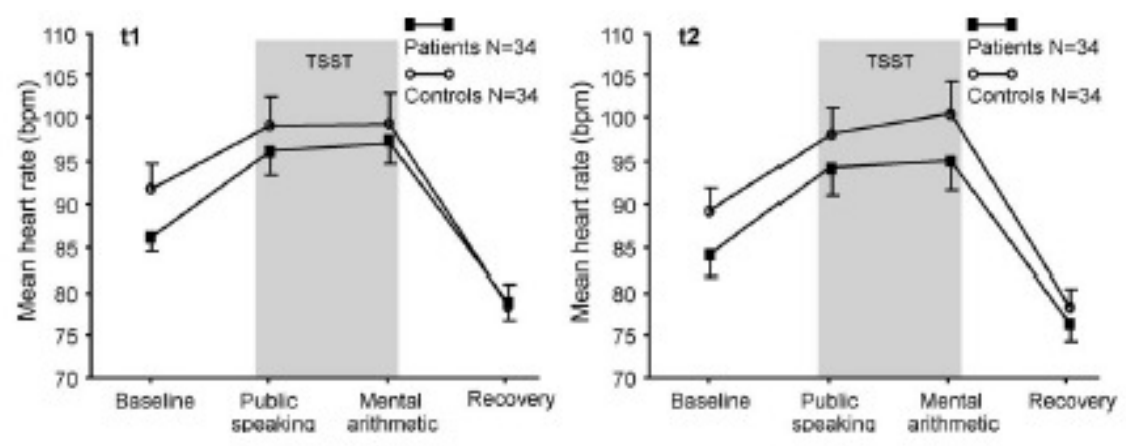

Figure 3 Mean heart rate in response to psychosocial stress (TSST) in patients with panic disorder and healthy control sub jects on 2 consecutive days $(\mathrm{t} 1, \mathrm{t} 2)$.

\section{Role of funding source}

Funding for this study was provided by the Medical Faculty of Dresden University of Technology (MedDrive); the Dresden University of Technology had no further role in study design; in the collection, analysis and interpretation of data; in the writing of the report; and in the decision to submit the paper for publication.

\section{Conflict of interest}

The authors of this study declare no conflicts of interest.

\section{Acknowledgments}

The authors thank the Medical Faculty of Dresden University of Technology for supporting this study with a Young Researcher grant (MedDrive).

\section{References}

- Abelson, J.L., Khan, S., Liberzon, I., Young, E.A., 2007. HPA axis activity in patients with panic disorder: review and synthesis of four studies. Depress Anxiety 24, 66-76.

- Albert, C.M., Chae, C.U., Rexrode, K.M., Manson, J.E., Kawachi, I., 2005. Phobic anxiety and risk of coronary heart disease and sudden cardiac death among women. Circulation 111, $480-487$.

- American Psychiatric Association, 2004. Diagnostic and statistical manual of mental disorders, fourth edition (DSM-IV-TR). Washington, DC: American Psychiatric Association. At risk for panic disorder. Biol. Psychiatry 57, 1485-1492.

- Bandelow, B., 1979. Panik- und Agoraphobieskala (PAS). Hogrefe, Göttingen/Bern/Toronto/ Seattle.

- Bandelow, B., Wedekind, D., Pauls, J., Broocks, A., Hajak, G., Rüther, E., 2000. Salivary cortisol in panic attacks. Am. J. Psychiatry 157, 454- 456.

- Beck, A.T., Ward, C.H., Medelson, M., Mock, J., Erbaugh, J., 1961. An inventory for measuring depression. Arch. Gen. Psychiatry 4, 561-571.

- Cameron, O.G., Lee, M.A., Curtis, G.C., McCann, D.S., 1987. Endocrine and physiological changes during ,spontaneous“ panic attacks. Psychoneuroendocrinology 12, 321-331.

- Derogatis, L.R., 1977. SCL-90-R, administration, scoring \& procedures manual-I for the R(evised) version. John Hopkins University School of Medicine, Baltimore. 
- Dickerson, S.S., Kemeny, M.E., 2004. Acute stressors and cortisol responses: a theoretical integration and synthesis of laboratory research. Psychol. Bull. 130, 355-391.

- Domschke, K., Ohrmann, P., Braun, M., Suslow, T., Bauer, J., Hohoff, C., Kersting, A., Engelien, A., Arolt, V., Heindel, W., Deckert, J., Kugel, H., 2008. Influence of the catecholOmethyltransferase val158met genotype on amygdala and prefrontal cortex emotional processing in panic disorder. Biol. Psychiatry 163, 13-20.

- Erhardt, A., Ising, M., Unschuld, P.G., Kern, N., Lucae, S., Puetz, B., Uhr, M., Binder, E.B., Holsboer, F., Kneck, M.E., 2006. Regulation of the hypothalamic-pituitary-adrenocortical system in patients with panic disorder. Neuropsychopharmacology 32, 2516-2522.

- Franke, G.H., 2002. SCL-90-R: symptom-checkliste. In: von Derogatis, L.R. (Ed.), Deutsche Version - Manual. Belts Test GmbH, Göttingen.

- Fries, E., Dettenborn, L., Kirschbaum, C., 2008. The cortisol awakening response (CAR): facts and future directions. Int. J. Psychophysiol. 30.

- Garcia-Leal, C., Parente, A.C., Del-Ben, C.M., Guimarães, F.S., Moreira, A.C., Elias, L.L., Graeff, F.G., 2005. Anxiety and salivary cortisol in symptomatic and nonsymptomatic panic patients and healthy volunteers performing simulated public speaking. Psychiatry Res. 133, $239-252$.

- Greenberg, P.E., Sisitsky, T., Kessler, R.C., Finkelstein, S.N., Berndt, E.R., Davidson, J.R.T., Ballenger, J.C., Fyer, Abby, J., 1999. The economic burden of the anxiety patients of the, 1990s. J. Clin. Psychiatry 7, 427-435.

- Gurguis, G.N., Mefford, I.N., Uhde, T.W., 1991. Hypothalamic-pituitary-adrenocortical activity in panic disorder: Relationship to plasma catecholamine metabolites. Biol. Psychiatry 30, 502-506.

- Hautzinger, M., Bailer, M., Worall, H., Keller, F., 1994. Beck-Depressions-Inventar (BDI), Bern: Huber.

- Hellhammer, D.H., Wüst, S., Kudielka, B., 2009. Salivary cortisol as a biomarker in stress research. Psychoneuroendocrinology 34, 163-171.

- Hoehn, T., Braune, S., Scheibe, G., Albus, M., 1997. Physiological, biochemical and subjective parameters in anxiety patients with panic disorder during stress exposure as compared with healthy controls. Eur. Arch Psychiatry Clin. Neurosci. 247, 264-274.

- Holsboer, F., von Bardeleben, U., Buller, R., Heuser, I., Steiger, A., 1987. Stimulation response to corticotropin-releasing hormone $(\mathrm{CRH})$ in patients with depression, alcoholism and panic disorder. Horm. Metab. Res. Suppl. 16, 80-88.

- Kern, S., Oakes, T.R., Stone, C.K., McAuliff, E.M., Kirschbaum, C., Davidson, R.J., 2008. Glucose metabolic changes in the prefrontal cortex are associated with HPA axis response to a psychosocial stressor. Psychoneuroendocrinology 33, 517-529.

- Kirschbaum, C., Pirke, K.M., Hellhammer, D.H., 1993. The „Trier Social Stress Test“ — a tool for investigating psychobiological stress responses in a laboratory setting. Neuropsychobiology 28, 76-81.

- Kirschbaum, C., Pruessner, J.C., Stone, A.A., Federenko, I., Gaab, J., Lintz, D., Schommer, N., Hellhammer, D.H., 1995. Persistent high cortisol responses to repeated psychological stress in a subpopulation of healthy men. Psychosom. Med. 57, 468 - 474.

- Kudielka, B.M., Hellhammer, D.H., Kirschbaum, C., 2007. Ten years of research with the trier social stress test—revisited. In: Harmon-Jones, E., Winkielman, P. (Eds.), Social Neuroscience: Integrating Biological and Psychological Explanations of Social Behavior. Guilford Press, New York, NY, US, pp. 56-83.

- Leyton, M., Be'langer, C., Martial, J., Beaulieu, S., 1996. Cardiovascular, neuroendocrine, and Monoaminergic responses to psychological stressors: Possible differences between remitted panic disorder patients and healthy controls. Biol. Psychiatry 5, 353-360.

- Mason, J.W., 1968. A review of psychoendocrine research on the pituitary-adrenal cortical system. Psychosom. Med. 30, 576-607.

- McEwen, B.S., 1998. Protective and damaging effects of stress mediators. N. Engl. J. Med. $338,171-179$. 
- $\quad$ Roy-Byrne, P.P., Uhde, T.W., Post, R.M., Gallucci, W., Chrousos, G.P., Gold, P.W., 1986. The corticotropin- releasing hormone stimulation test in patients with panic disorder. Am. J. Psychiatry 143,896-899.

- Schmidt-Reinwald, A., Pruessner, J.C., Hellhammer, D.H., Federenko, I., Rohleder, N., Schürmeyer, T.H., Kirschbaum, C., 1999. The cortisol response to awakening in relation to different challenge tests and a 12-hour cortisol rhythm. Life Sci. 64, 1653-1660.

- Schommer, N.C., Hellhammer, D.H., Kirschbaum, C., 2003. Dissociation between reactivity of the hypothalamus-pituitary-adrenal axis and the sympathetic-adrenal-medullary system to repeated psychosocial stress. Psychosom. Med. 65, 450— 460.

- Schreiber, W., Lauer, C.J., Krumrey, K., Holsboer, F., Krieg, J.C., 1996. Dysregulation of the hypothalamic-pituitary-adrenocortical system in panic disorder. Neuropsychopharmacology $15,7-15$.

- Smoller, J.W., Gardner-Schuster, E., Covino, J., 2008. The genetic basis of panic and phobic anxiety disorders. Am. J. Med. Genet. Part C Semin. Med. Genet. 148C, 118-126.

- Smoller, J.W., Yamaki, L.H., Fagerness, J.A., Biederman, J., Racette, S., Laird, N.M., Kagan, J., Snidman, N., Faraone, S.V., Hirshfeld-Becker, D., Tsuang, M.T., Slaugenhaupt, S.A., Rosenbaum, J.F., Sklar, P.B., 2005. The corticotropin-releasing hormone gene and behavioral inhibition in children. Biol. Psychiatry 57, 1485-1492.

- $\quad$ Spitzer, R.L., Williams, J.B.W., Gibon, M., First, M., 1990. SCID: User's Guide for the Structured Clinical Interview for DSM-III-R. American Psychiatric Press, Washington DC.

- Van Cauter, E., Refetoff, S., 1985. Evidence for two subtypes of Cushing's disease based on the analysis of episodic cortisol secretion. N. Engl. J. Med. 23, 1343-1349.

- Wang, J., Rao, H., Wetmore, G.S., Furlan, P.M., Korczykowski, M., Dinges, D.F., Detre, J.A., 2005. Perfusion functional MRI reveals cerebral blood flow pattern under psychological stress. Proc. Natl. Acad. Sci. USA 102, 17804-17809.

- Wittchen, H.U., Zaudis, M., Schramm, E., Spengler, P., Mombour, W., Klug, J., Horn, R., 1990. Strukturiertes Klinisches Interview für DSM-III-R. Beltz Test-Gesellschaft, Weinheim. 